Corresponding Author: ELhadi M.Awooda; Consultant in Restorative Dentistry and Health Management email: dr.alhadi@yahoo.com

Received 12 November 2020 Accepted 9 December 2020 Published 31 December 2020

Production and Hosting by Knowledge E

(c) Elhadi M. Awooda and Reem E. Siddig. This article is distributed under the terms of the Creative Commons

Attribution License, which permits unrestricted use and redistribution provided that the original author and source are credited.

Editor-in-Chief:

Prof. Mohammad A. M. Ibnouf

\section{Tele Screening, Triaging of Dental Patients, And Reopening of Dental Services During SAR-CoV2 Pandemic}

\author{
Elhadi M. Awooda' and Reem E. Siddig ${ }^{1}$ \\ ${ }^{1}$ Consultant in Restorative Dentistry and Health Management \\ ${ }^{2}$ Researcher at Sudanese Medical Sciences Research Association (SMRA)
}

\section{Abstract}

Background: The nature of dental profession requires a dentist to be in a close proximity to the patient's mouth to perform various operative treatments including procedures such as generating aerosols. As such, dentistry is considered as one of the riskiest profession for the cross-transmission of SARS-CoV2. Although the prevalence and incidence of SARS-CoV2 continue to increase worldwide; the dental services and treatment of non-emergency cases have resumed in many countries including Sudan. Moreover, the use of smartphone technology in communication with patients can play an important role in controlling the spread of SARS-CoV2. Therefore, the aim of this short review was to draw a summary on using smartphone technology for telescreening dental patients prior to their visit to the clinics and to set guidelines and rules for dental treatment during SARS-CoV2 pandemic.

Methods: For the purpose of this study, telescreening is referred as a virtual contact between patients and healthcare personnel used to provide health services during the highly contiguous SARS-CoV2 pandemic. Using a smartphone, dental patients can be screened and questioned about their medical history and particularly for SARS-CoV2 (for symptoms such as fever, headache, coughing, breath shortness, loss of smell or taste). They should also be asked about their travel history to epidemic area, visiting hospital or coming in contact with a confirmed SARS-CoV2 patient during the last 14 days. On arrival to a dental clinic, the patient's temperature should be measured, instruction for wearing masks, hand hygiene, and keeping distances from others inside the waiting zone.

Conclusion: Telescreening and triaging will minimize the time of patients' exposure to dental clinic environments, hence decreasing the risk of getting SARS-CoV2 infection for both patients and dental healthcare workers.

Keywords: COVID-19, dentistry, procedure generating aerosols, smartphone, social distance, surgical masks.

\section{Introduction}

The global SARS-CoV2 pandemic continues to challenge scientists around the world on discovering its mode of transmission, treatments, and vaccination. As it continues spreading worldwide, health services resources, healthcare professions, economies, 
and social life around world remain under the immense pressure of depletion, depression, and constraints on the future of earth's planet life [1]. The rapid spread of the SARS-CoV2 highlights the possibility of airborne transmission through aerosols and droplets [2]. A contradictory result has been questioned on the risk of getting crossinfection to healthcare workers (HCWs) during their routine duties, as an early Chinese study reported increased infection rates among $\mathrm{HCWs}$, while a retrospective study from Switzerland [3] was inconsistent to others and revealed that cross-infection of SARSCoV2 clusters occurred within the same household or after an enclosed, extended close contact $[4,5]$. Dentistry is one of the medical professions that involves "procedures generating aerosols" (PGA) and represents one of the high-risk professions in crosstransmission of SARS-CoV2 [6], to both patients and HCWs [7].

Dentistry has been recommended as a non-emergency treatment until the pandemic is controlled. However, at the same time, dental and oral diseases are chronic in nature; if not managed early, the consequences may cost time, resources, efforts, and finances. Likewise, many other non-emergency treatments examples: early dental caries of the enamel and dentin, gingivitis or replacement of a missing tooth or teeth) are of paramount importance to the patient's psychological, physiological, physical, and mental well-being. Many countries have recently implemented strategies for the reopening of dental services [8]. In Sudan, however, the clinics were re-opened on June 10, 2020. Certain guidelines are required to be followed to avoid risking the dental health care workers' (DHCPs), patients', or community cross-infection of the SARS-CoV2.

The objective of this short communication was to discuss the implementation of telescreening and triage in dental clinics to prevent and control the spread SARS-CoV2 and to set guidelines and regulations inside the clinics for patients seeking dental treatment during this pandemic.

\section{Methods}

This report represents a summary of the experiences and recommendations of other studies from the literature reviewed for using tele-screening and triage during the SARSCoV2 pandemic and reopening of dental services.

Tele-screening: Announcements through different social media and communication systems to advise patients; to use the tele-communication for consultation and arranging an appointment. One of the most practical and easily available option is using a smartphone, and when calling, the patient should answer the following questions:

1. Have you traveled to any area with a high incidence of SARS-CoV2, abroad or inside the country $[6,9]$ ?

2. Have you visited or met anyone who within the last two months has traveled to any area with the SARS-CoV2 epidemic?

3. Have you come in contact with a known case of SARS-CoV2 or a suspicious one, at home, markets, neighborhood, social events, etc.? 
4. Have you taken any healthcare services (primary, secondary, or tertiary) during the last two weeks for whatever reason (sick, accompanied, visitor, taxi driver or $\mathrm{HCW})$ ?

5. Have you experienced a fever or headache in the past days?

6. Have you experienced dry cough or shortness of breath during the past few days? $[6,9]$

7. Have you contacted people in public, work, meetings, social events during the past few days? [10]

8. Have you lost your sense of smell or taste during the last two weeks? [11]

9. Have you heard or seen two or more people with fever and/or respiratory symptoms at home, office, school classrooms, etc. within the last two weeks?

\subsection{Triaging on arrival to the dental clinic}

1. The entrance doors should be kept open in case of non-sliding doors.

2. Assigned trained nurse or junior dentist should act as an infection control officer, to be responsible for infection control and prevention measures, for patients and for other dental personals.

3. Post signs at entrances reminding patients that the disease has not gone yet and labeled instructions to wear masks, keeping a distance from others inside the waiting zone, no handshaking, hand hygiene, and using disposable tissues while coughing or sneezing $[12,13]$.

4. Inform patients verbally or through a written brochure about the protocol the clinic follows to prevent and control the SARS-CoV2, including instructions about compulsory temperature measurement, wearing masks, hand hygiene, and keeping a distance from others.

5. The patient has the right to refuse following the guidelines and rules set by the clinic/hospital, but they should have the clinic/hospital compound and not be allowed to enter. In case they accept the rules and guidelines, a verbal or signed written consent is mandatory.

6. Before starting any procedure, the patient's temperature should be measured $[6,9]$ and the presence of any respiratory tract symptoms be assessed [14].

7. An alcoholic disinfectant and masks should be available to all individuals inside the waiting room and should be used all the time $[14,15]$

8. Instructions should be given to the patient and/or co-patients about hand hygiene, either by the use of hand sensitizer or hand washing [14]

9. No-touch receptacles should be provided for the disposal of tissues [11, 13, 15]. 
10. The clinic should have enough space to maintain a physical distance of minimum $1.5 \mathrm{~mm}$ between two people $[13,16]$.

11. Questions about patient's medical history that was previously asked through telephone should be asked again.

12. A history of the chief complaint should be carried out in a triage area near the entrance.

13. For suspected/confirmed cases of SARS-CoV2 that are medically stable, authorities responsible for further investigation and management should be consulted [14].

14. To prevent cross-infection of SARS-CoV2 within the dental clinics, for cases in (xiii) the treatment should be rescheduled for after a minimum of two weeks, and the patient should be encouraged to self-quarantine $[10,13,16]$.

15. Cases with clear acute symptoms and signs of SARS-CoV2 should be referred to a nearby hospital for further investigations and management.

16. If emergency treatment is needed for those patients with suspicion/confirmed SARS-CoV2, appropriate personal protective equipment should be used and the protocols of infection control prevention for highly contagious airborne viruses be followed [13].

\section{Discussion}

Although, in Sudan, the lockdown strategy was implemented for more than three months, the SARS-CoV2 continues to spread her and all over the world, with no near-future resolution of this pandemic. Diseases of dental and oral cavity origins are most likely chronic in nature and early intervention gives better treatment outcomes and prevent unwanted consequences. During the lockdown period, many dental and oral diseases may have been developed, including the highly prevalent dental caries, gingivitis, and periodontitis. The reopening is an inevitable decision for the prevention and treatment of oral and dental diseases.

Before the reopening, employers and DHCPs must understand the following key points: dental settings have unique characteristics that warrant specific infection control consideration; prioritizing the most critical dental services and providing care in an important way that minimizes harm to patients from delaying care and harm to personnel from potential exposure to SARS-CoV2. Appropriate training should be given to all DHCWs on infection prevention control measures. Also, clinic attendees should have enough knowledge and awareness about SARS-CoV2 symptoms and its mode of transmission and prevention. Audiovisuals, verbal, and written instructions (on coughing and sneezing etiquette, proper hand hygiene, wearing mask all the time, and keeping distances from other while waiting) should be provided to remind and encourage patients to prevent the cross-transmission of the disease.

Tele-screening can be used as the first step in providing dental consultation and treatments. One of the recommendations is to communicate with patients via smartphones. This minimizes patients' attendance to the clinic. It also reduces the waiting 
time, eventually preventing unwanted crowding. Also, patients can be advised to defer the treatment for the coming weeks or even be referred for further medical consultation in case of any suspicious of SARS-CoV2 infection.

Recent advances in communication technology (e.g., webinars, video calling, Skype, etc.) paired with high internet speed and easy-to-use and highly capable smartphones make it easy for patient and healthcare provider to communicate virtually and to draw treatment planning [7]. American Dental Association (ADA) recommends tele-dentistry as a mean for patient care through the following modalities: 1) Synchronous (live video); means direct interaction between patient and DHCW using audiovisual communication. 2) Asynchronous (store and forward); for example, radiographs, photographs, video, digital impressions and photomicrographs of patients, to evaluate their dental conditions and arrangement for the suitable alternative way of management. 3) Remote patient monitoring (RPM); it means collecting personal health data of an individual in one location through electronic communication to a dental practitioner who use the information outside the real time or live interaction to set plan for management. 4) Mobile health (mHealth); through uses of smart phones to provide dental health care and information. [17]

Worldwide, many hospitals and clinics including the different branches and specialties in medicine and dentistry have recently implemented tele-screening. However, barriers to broad implementation of such services continue to exist in many countries including Sudan. Where the tele-communication infrastructures still lack and majority of the people reside in rural areas, with electricity deficiency, inability to afford smart phones, and incapability to use it. Still with the limited facilities of smart technology in Sudan, it can provide a minimal of dental consultation to avoid risking patients cross-infection from health care facilities. Recent policy changes promote the use of tele-screening as a way to deliver acute, chronic, primary, and specialty care [18]. In addition, the cost of protective equipment and dental equipment, provider training, and payment regulatory services must be taken into account [19].

Tele-screening in dentistry may have some potential limitations, because dental treatment is an interventional operative procedure that necessitates the presence of operator, instruments, and artificial biomaterials. Another issue is the level of comfort with technology for DHCPs and patients because some patients may have privacy concerns, so the issue of consent should be considered [8]. Moreover, the cultural acceptance of conducting virtual visits instead of in-person visits may need time.

Another guideline is patients' triaging on arrival to the clinic, which assists in determining the acuity of concerns necessitating early intervention as well as a decrease in overutilization of healthcare resources and particularly for the prevention of crossinfection of SARS-CoV2.

\section{Conclusion}

Many countries have recently implemented strategies for the reopening of dental services, it is state of a new normal which cannot be as normal as practiced in the past before the SARS-CoV2 pandemic. There is a need to avoid crowding, social distancing, 
hand hygiene, minimizing the waiting time of patients inside clinics, and appropriate PPE.

Providing dental treatment services effectively and safely for both patients and DHCWs can be guaranteed through tele-screening and triage implementation.

\section{Authors' contributions}

This work was carried out by Elhadi Mohieldin Awooda (EMA) \& Reem Isam Siddig (RIS), where EMA who designed the study, reviewed the literature, wrote the protocol, and revised the manuscript critically for important intellectual content. RIS, wrote the draft, discussion, and literature searched. Both authors, read and approved the final manuscript.

\section{Competing Interests}

Both authors have declared that no competing interests exist.

\section{Ethics approval:}

The information provided in this manuscript is of the author's and coauthor own opinion. The recommendations and summary discussed were extracted from peer review journals and pertinent literatures, were cited accordingly.

\section{Consent for publication:}

Not applicable.

\section{Availability of data and materials:}

All relevant data were within the manuscript, in case of any queries, contact the correspondence author, EMA.

\section{References}

[1] Zou, L., Ruan, F., Huang, M., et al. (2020). SARS-CoV-2 viral load in upper respiratory specimens of infected patients. The New England Journal of Medicine, vol. 382, no. 12, pp. 1177-1179.

[2] Chan, J. F., Yuan, S., Kok, K. H., et al. (2020). A familial cluster of pneumonia associated with the 2019 novel coronavirus indicating person-to-person transmission: a study of a family cluster. Lancet, vol. 395, no. 10223 , pp. 514-523.

[3] Veraa, C., Heidia, L., Janb, P., et al. (2020). Transmission risk of SARS-CoV-2 to healthcare workers - observational results of a primary care hospital contact tracing. Swiss Medical Weekly, vol. 150, p. w20257. doi:10.4414/smw.2020.20257

[4] Rothe, C., Schunk, M., Sothmann, P., et al. (2020). Transmission of 2019-nCoV infection from an asymptomatic contact in Germany. The New England Journal of Medicine, vol. 382, no. 10, pp. 970-971. Retrieved from: http://dx.doi.org/10.1056/NEJMc2001468 
[5] Liu, Y., Eggo, R. M., and Kucharski, A. J. (2020). Secondary attack rate and super spreading events for SARS-CoV-2. Lancet, vol. 395, no. 10227, p. E47: doi: http://dx.doi.org/10.1016/S0140-6736(20)30462-1

[6] Peng, X., Xu, X., Li, Y., et al. (2020). Transmission routes of 2019-nCoV and controls in dental practice. International Journal of Oral Science, vol. 12, no. 9, PP.1-6. Retrieved from: https://doi.org/ 10.1038/s41368-020-0075-9.

[7] Tom, S. and Jessyka, G. (2020). Telemedicine in the era of the COVID-19 pandemic: implications in facial plastic surgery. Facial Plastic Surgery \& Aesthetic Medicine, vol. 22, no. 3, pp. 155-156. doi:10.1089/fpsam.2020.0163

[8] Centers for Disease Control and Prevention. USA (2020). CDC Releases Interim Reopening Guidance for Dental Settings. Division of Oral Health, National Center for Chronic Disease Prevention and Health Promotion. Retrieved from: https://www.cdc.gov/oralhealth/infectioncontrol/statement-COVID.html

[9] Cantore, S. and Ballini, A. (2020). Coronavirus disease 2019 (COVID-19) pandemic burst and its relevant consequences in dental practice. The Open Dentistry Journal, vol. 14, pp. 111-112. Retrieved from: https://doi.org/10.2174/1874210602014010111

[10] Ather, A., Patel, B., Ruparel, N., et al. (2020). Corona Virus Disease 19 (COVID-19): Implication for Clinical Dental Care. Journal of Endodontics, vol. 46, no. 5, pp. 584-595.

[11] Spinato, G., Fabbris, C., Polesel, J., et al. (2020). Alterations in smell or taste in mildly symptomatic outpatients with SARS-CoV-2 infection. JAMA, vol. 323, no. 20, pp. 2089-2090. doi:10.1001/jama.2020.6771

[12] Centers for Disease Control and Prevention (CDC). USA (2020). Strategies to Prevent the Spread of COVID-19 in Long Term Care Facilities (LTCF) 2020. Retrieved from: https://www.cdc.gov/coronavirus/ 2019-ncov/healthcare-facilities/prevent-spread-in-long-term-carefacilities.html

[13] Fallahi, H., Kehyan, S., Zandian, D., et al. (2020). Being a front line dentist during the COVID-19 Pandemic: a literature review. Maxillofacial Plastic and Reconstructive Surgery, vol. 42, no. 1, p. 12. doi:10.1186/s40902-020-00256-5

[14] Ge, Z., Yang, L., Xia, J., et al. (2020). Possible aerosol transmission of COVID-19 and special precautions in dentistry. Journal of Zhejiang University Science B, vol. 21, no. 5, pp. 361368. doi:10.1631/jzus.b2010010.

[15] Spagnuolo, G., De Vito, D., Rengo, S., et al. (2020). COVID-19 outbreak: an overview on dentistry. International Journal of Environmental Research and Public Health, vol. 17, no. 6, p. 2094. doi:10.3390/ijerph17062094

[16] Klyn, S. L., Cummings, D. E., Richardson, B. W., et al. (2001). Reduction of bacteria-containing spray produced during ultrasonic scaling. General Dentistry, vol. 49, no. 6, pp. 648-652.

[17] American Dental Association (ADA) Policy on Teledentistry. https://www.ada.org/en/about-the-ada/ ada-positions-policies-and-statements/statement-on-teledentistry. (accessed December 2020).

[18] Bashushur, R., Doarn, C. R., Frenk, J. M., et al. (2020). Telemedicine and the COVID19 pandemic, lessons for the Future. Telemedicine Journal and e-Health, vol. 26, no. 5, pp. 571-573.

[19] Hollander, J. E. and Carr, B. G. (2020). Virtually perfect? Telemedicine for Covid-19. The New England Journal of Medicine, vol. 382, pp. 1679-1681. doi:10.1056/NEJMp2003539 